UDC: 004.93

\title{
A hybrid regularizers approach based model for restoring image corrupted by Poisson noise
}

\author{
Thi Thu Thao Tran', Cong Thang Pham ${ }^{2, a}$ \\ ${ }^{1}$ The University of Danang - University of Economics, \\ 71 Ngu Hanh Son st., Danang, 550000, Vietnam \\ ${ }^{2}$ The University of Danang - University of Science and Technology, \\ 54 Nguyen Luong Bang st., Danang, 550000, Vietnam \\ E-mail: ${ }^{a}$ pcthang@dut.udn.vn
}

Received 26.05.2021, after completion - 22.07.2021. Accepted for publication 06.08.2021.

Image denoising is one of the fundamental problems in digital image processing. This problem usually refers to the reconstruction of an image from an observed image degraded by noise. There are many factors that cause this degradation such as transceiver equipment, or environmental influences, etc. In order to obtain higher quality images, many methods have been proposed for image denoising problem. Most image denoising method are based on total variation (TV) regularization to develop efficient algorithms for solving the related optimization problem. TV-based models have become a standard technique in image restoration with the ability to preserve image sharpness.

In this paper, we focus on Poisson noise usually appearing in photon-counting devices. We propose an effective regularization model based on combination of first-order and fractional-order total variation for image reconstruction corrupted by Poisson noise. The proposed model allows us to eliminate noise while edge preserving. An efficient alternating minimization algorithm is employed to solve the optimization problem. Finally, provided numerical results show that our proposed model can preserve more details and get higher image visual quality than recent state-of-the-art methods.

Keywords: image denoising, total variation, minimization, Poisson noise

Citation: Computer Research and Modeling, 2021, vol. 13, no. 5, pp. 965-978.

Pham Cong Thang (corresponding author) would like to express his sincere gratitude to colleagues at IT Faculty, DUT, for helpful comments. The authors also thank reviewers and Editors for their insightful comments and suggestions. This work is supported by The Murata Science Foundation and The University of Danang-University of Science and Technology, code number of Project T2020-02-07MSF. 


\section{Introduction}

Image denoising is one of popular problems in digital image processing and. In receiving and transmitting process, the image is always degraded by noise [Pham, 2015; Pham, 2018a]. In photoncounting devices such as radiography, astronomical imaging, electronic microscopy... [Bardsley, 2011; Frosioa, 2009; Sarder, 2006; Wang, 2013], images may be corrupted by noise. In this case, the noise is often referred to as Poisson noise. The Poisson component accounts for the signal-dependent uncertainty inherent to the photon counting process [Lanza, 2014; Li, 2015; Pham, 2018b]. As is well known, several approaches have been developed for recovering images corrupted by the Poisson noise. Among them, one of popular approaches for Poisson noise removal is perhaps total variation model (TV) using the TV norm as regularization term, formulated as follows [Le, 2007]:

$$
u^{*}=\underset{u}{\arg \min }\left(\int_{\Omega}|\nabla u| d x+\beta \int_{\Omega}(u-f \log u) d x\right),
$$

where the reconstructed image $u^{*}$ is computed from the observed image $f=f(x)$ with $x=\left(x_{1}, x_{2}\right) \in$ $\in \Omega, \Omega \subseteq \mathbb{R}^{2}$ being an open bounded domain; $u$ must be positive almost everywhere over $\Omega$; $\beta$ are positive regularization parameter; $\nabla u$ stands for first order total variation of $u$ defined later in (4).

As is well known, the TV regularizer framework causes the numerous artifact effects in the recovered results, such that small details filtered out with noise in the process of denoising, and translation of smooth regions into piecewise constant regions [Zhang, 2012; Khan, 2017]. To avoid this problem, many regularization techniques for the denoising problem have been introduced by replacing the TV norm by a high-order TV norm, such that second order total variation [Lysaker, 2003; Zhang, 2019; Pham, 2021], non-local total variation [Kayyar, 2018], Euler's elastic model [Zhang, 2017], a mean curvature model [Zhu, 2014; Myllykoski, 2015], total generalized variation [Bredies, 2010; Liu, 2019]. Besides, hybrid regularizers models for image denoising are also introduced by combining a higher-order regularizer with the TV norm [Chan, 2015; Jiang, 2013; Papafitsoros, 2014; Yang, 2019].

Recently, fractional-order derivatives are widely applied in image processing [Zhang, 2015; Chen, 2015; Chowdhury, 2020]. The works have reflected good performance of the fractional-order derivative in image denoising with edge preserving. Authors in [Chowdhury, 2020] proposed the fractional-order total variation model (FTV) for Poisson image denoising as follows:

$$
u^{*}=\underset{u}{\arg \min } \int_{\Omega}\left|\nabla u^{\alpha}\right| d x+\beta \int_{\Omega}(u-f \log u) d x,
$$

where $\beta$ are positive parameters; $\nabla u^{\alpha}$ stands for fractional-order total variation defined in (5).

Motivated by the previous works, we propose a Poisson noise removal model that can substantially reduce artifact effects while preserving edges in the restored images. The proposed model is designed by combining advantages of the first-order (1) total variation and fractional-order total variation total (2). We extend an efficient alternating minimization method for solving the optimization problem. Furthermore, we provide experimental results to demonstrate the efficiency of our algorithm for considered problem, in comparison with state-of-the-art methods.

This paper is organized as follows. In section 2 , we briefly describe the proposed model. We establish the proposed algorithm for solving the optimization problem in section 3. In section 4, numerical experiments to illustrate the outstanding performance of our approach are provided. Finally, we make conclusions of this work in section 5 . 


\section{The proposed model for Poisson noise removal}

In this paper, we consider to modify the total variation model (1) by adding a fractional-order functional for restoring Poissonian images. It leads to the following minimization problem:

$$
\begin{gathered}
u^{*}=\underset{u}{\arg \min } E(u), \\
E(u)=\gamma_{1} \int_{\Omega}|\nabla u| d x+\gamma_{2} \int_{\Omega}\left|\nabla u^{\alpha}\right| d x+\beta \int_{\Omega}(u-f \log u) d x,
\end{gathered}
$$

where $\gamma_{1}, \gamma_{2}$, and $\beta$ are positive parameters; $\Omega \subset \mathbb{R}^{2}$ be bounded open set and $u$ must be positive almost everywhere over $\Omega ; \nabla u^{\alpha}$ stands for fractional-order total variation defined in (5).

The concept of the first-order and fractional-order total variation can be found in [Aubert, 2006; Zhang, 2015; Chen, 2015; Dong, 2016]. The discrete gradient $\nabla$ of an image $u$ for the pixel location $(i, j)$ in $u(i=1, \ldots, M ; j=1, \ldots, N)$ is defined as follows:

$$
\begin{gathered}
\nabla_{1} u_{i, j}=u_{i+1, j}-u_{i, j}, \quad \nabla_{2} u_{i, j}=u_{i, j+1}-u_{i, j}, \\
\nabla u_{i, j}=\left(\nabla_{1} u_{i, j}, \nabla_{2} u_{i, j}\right), \quad\left|\nabla u_{i, j}\right|=\sqrt{\left(\nabla_{1} u_{i, j}\right)^{2}+\left(\nabla_{2} u_{i, j}\right)^{2}} .
\end{gathered}
$$

Due to the convenience in numerical implementation, from Greunwald-Letnikov (GL) fractional-order derivative, the discrete form of the fractional-order gradient $\nabla^{\alpha}$ can be evaluated by [Zhang, 2012]:

$$
\nabla^{\alpha} u=\left[\nabla_{1}^{\alpha} u, \nabla_{2}^{\alpha} u\right],
$$

where the discrete gradients $\nabla_{1}^{\alpha} u$ and $\nabla_{2}^{\alpha} u$ are defined as follows:

$$
\left(\nabla_{1}^{\alpha} u\right)_{i, j}=\sum_{k=0}^{K-1} C_{k}^{\alpha} u_{i-k, j}, \quad\left(\nabla_{2}^{\alpha} u\right)_{i, j}=\sum_{k=0}^{K-1} C_{k}^{\alpha} u_{i, j-k} .
$$

Parameter $K$ is the number of neighboring pixels that are used to compute the fractional-order derivative at each pixel; the coefficients $\left\{C_{k}^{\alpha}\right\}$ are defined as follows:

$$
C_{k}^{\alpha}=\frac{\Gamma(\alpha+1)}{\Gamma(k+1) \Gamma(\alpha+1-k)}, \quad k=1, \ldots, K-1 .
$$

The discrete fractional-order total variation $\nabla^{\alpha}$ of $u \in \Omega$ is defined as follows:

$$
\left|\nabla^{\alpha} u\right|=\sqrt{\left(\nabla_{1}^{\alpha} u_{i, j}\right)^{2}+\left(\nabla_{2}^{\alpha} u_{i, j}\right)^{2}} .
$$

\section{Computational method}

In literature, we can find many efficient algorithms for solving the TV-regularized Poison denoising model (3), such as a primal-dual algorithm [Chambolle, 2004; Pham, 2019], an augmented Lagrangian method [He, 2014; Huang, 2008; Wang, 2008; He, 2014], the split Bregman method [Setzer, 2010; Goldstein, 2009; Chen, 2015; Pham, 2020], etc.

In this paper, we decide to employ the alternating optimization method to solve the considered optimization problem. In this section, we derive the numerical method for problem (3) in detail. Referring to the variable splitting method [Huang, 2008; He, 2014; Goldstein, 2009; Chen, 2015], 
we introduce two new variables $(\vartheta, \omega)$ and rewrite (3) in the constrained optimization problem as follows:

$$
\begin{gathered}
\min _{\vartheta, \omega, u}\left(\gamma_{1}\|\vartheta\|_{1}+\gamma_{2}\|\omega\|_{1}+\beta\langle\mathbf{1}, u-f \log u\rangle\right), \\
\text { s.t. } \vartheta=\nabla u, \omega=\nabla^{\alpha} u .
\end{gathered}
$$

The augmented Lagrangian functional for the constrained optimization problem (6) is defined as:

$$
\begin{aligned}
\min _{\vartheta, \omega, u, \xi_{1}, \xi_{2}}=\left(\gamma_{1}\|\vartheta\|_{1}+\gamma_{2}\|\omega\|_{1}+\beta\langle\mathbf{1}, u-f \log u\rangle\right. & -\left\langle\xi_{1}, \vartheta-\nabla u\right\rangle+\frac{\mu_{1}}{2}\|\vartheta-\nabla u\|_{2}^{2}- \\
& \left.-\left\langle\xi_{2}, \omega-\nabla^{\alpha} u\right\rangle+\frac{\mu_{2}}{2}\left\|\omega-\nabla^{\alpha} u\right\|_{2}^{2}\right),
\end{aligned}
$$

where $\mu_{1}, \mu_{2}$ - positive parameters; $\vartheta_{1}, \vartheta_{2}$ - Lagrangian multipliers.

The minimization method to solve the problem (7) can be expressed as follows:

$$
\left\{\begin{aligned}
u^{(k+1)}= & \underset{u}{\arg \min }\left(\beta\langle\mathbf{1}, u-f \log u\rangle-\left\langle\xi_{1}^{(k)}, \vartheta^{(k)}-\nabla u\right\rangle+\frac{\mu_{1}}{2}\left\|\vartheta^{(k)}-\nabla u\right\|_{2}^{2}-\right. \\
& \left.-\left\langle\xi_{2}^{(k)}, \omega^{(k)}-\nabla^{\alpha} u\right\rangle+\frac{\mu_{2}}{2}\left\|\omega^{(k)}-\nabla^{\alpha} u\right\|_{2}^{2}\right), \\
\vartheta^{(k+1)}= & \underset{\vartheta}{\arg \min }\left(\gamma_{1}\|\vartheta\|_{1}-\left\langle\xi_{1}^{(k)}, \vartheta-\nabla u^{(k+1)}\right\rangle+\frac{\mu_{1}}{2}\left\|\vartheta-\nabla u^{(k+1)}\right\|_{2}^{2}\right), \\
\omega^{(k+1)}= & \underset{\omega}{\arg \min }\left(\gamma_{2}\|\omega\|_{1}-\left\langle\xi_{2}^{(k)}, \omega-\nabla^{\alpha} u^{(k+1)}\right\rangle+\frac{\eta_{2}}{2}\left\|\omega-\nabla^{\alpha} u^{(k+1)}\right\|_{2}^{2}\right),
\end{aligned}\right.
$$

with update for $\xi_{1}^{(k+1)}, \xi_{2}^{(k+1)}$ :

$$
\left\{\begin{array}{l}
\xi_{1}^{(k+1)}=\xi_{1}^{(k)}+\mu_{1}\left(\nabla u^{(k+1)}-\vartheta^{(k+1)}\right), \\
\xi_{2}^{(k+1)}=\xi_{2}^{(k)}+\mu_{2}\left(\nabla^{\alpha} u^{(k+1)}-\omega^{(k+1)}\right) .
\end{array}\right.
$$

The $u$ subproblem in (8) is given by:

$$
\begin{aligned}
u^{(k+1)}= & \underset{u}{\arg \min }\left(\beta\langle\mathbf{1}, u-f \log u\rangle-\left\langle\xi_{1}^{(k)}, \vartheta^{(k)}-\nabla u\right\rangle+\frac{\mu_{1}}{2}\left\|\vartheta^{(k)}-\nabla u\right\|_{2}^{2}-\right. \\
& \left.-\left\langle\xi_{2}^{(k)}, \omega^{(k)}-\nabla^{\alpha} u\right\rangle+\frac{\mu_{2}}{2}\left\|\omega^{(k)}-\nabla^{\alpha} u\right\|_{2}^{2}\right)= \\
= & \frac{\mu_{1}}{2}\left\|\vartheta-\nabla u^{(k+1)}-\frac{\xi_{1}^{(k)}}{\mu_{1}}\right\|_{2}^{2}+\frac{\mu_{2}}{2}\left\|\omega-\nabla^{\alpha} u^{(k+1)}-\frac{\xi_{2}^{(k)}}{\mu_{2}}\right\|_{2}^{2}+\beta\langle\mathbf{1}, u-f \log u\rangle .
\end{aligned}
$$

Thus, we get:

$$
\mu_{1} \nabla^{T}\left(\nabla u+\frac{\xi_{1}^{(k)}}{\mu_{1}}-\vartheta^{(k)}\right)+\mu_{2}\left(\nabla^{\alpha}\right)^{T}\left(\nabla^{\alpha} u+\frac{\xi_{2}^{(k)}}{\mu_{2}}-\omega^{(k)}\right)+\beta \frac{u-f}{u}=0 .
$$

At the $k$-th iteration, we use the following discretization scheme:

$$
\begin{aligned}
\mu_{1} \nabla^{T} \nabla u^{(k+1)}+\mu_{1} \nabla^{T}\left(\frac{\xi_{1}^{(k)}}{\mu_{1}}-\vartheta^{(k)}\right)+\mu_{2}\left(\nabla^{\alpha}\right)^{T} \nabla^{\alpha} u^{(k+1)}+ & \\
& +\mu_{2}\left(\nabla^{\alpha}\right)^{T}\left(\frac{\xi_{2}^{(k)}}{\mu_{2}}-\omega^{(k)}\right)+\beta \frac{u^{(k+1)}-f}{u^{(k)}}=0 .
\end{aligned}
$$


Therefore, we have:

$$
\begin{aligned}
&\left(u ^ { ( k ) } \left(\mu_{1} \nabla^{T} \nabla u^{(k+1)}+\right.\right.\left.\left.\mu_{2}\left(\nabla^{\alpha}\right)^{T} \nabla^{\alpha}\right)+\beta\right) u^{(k+1)}= \\
&=\left(\mu_{1} \nabla^{T}\left(\vartheta^{(k)}-\frac{\xi_{1}^{(k)}}{\mu_{1}}\right)+\mu_{2}\left(\nabla^{\alpha}\right)^{T}\left(\omega^{(k)}-\frac{\xi_{2}^{(k)}}{\mu_{2}}\right)\right) u^{(k)}+\beta f .
\end{aligned}
$$

Under the periodic boundary conditions, $u^{(k+1)}$ in the system (10) can be efficiently solved by fast Fourier transform (FFT) [Wang, 2008] as follows:

$$
u^{(k+1)}=\mathcal{F}^{-1}\left(\frac{\mathcal{F}\left(\left(\mu_{1} \nabla^{T}\left(\vartheta^{(k)}-\frac{\xi_{1}^{(k)}}{\mu_{1}}\right)+\mu_{2}\left(\nabla^{\alpha}\right)^{T}\left(\omega^{(k)}-\frac{\xi_{2}^{(k)}}{\mu_{2}}\right)\right) u^{(k)}+\beta f\right)}{u^{(k)}\left(\mu_{1} \mathcal{F}\left(\nabla^{T} \nabla\right)+\mu_{2} \mathcal{F}\left(\left(\nabla^{\alpha}\right)^{T} \nabla^{\alpha}\right)\right)+\beta}\right),
$$

where $\mathcal{F}$ and $\mathcal{F}^{-1}$ are the forward and inverse Fourier transform operators.

The $\vartheta$ and $\omega$ subproblems are given by:

$$
\begin{aligned}
\vartheta^{(k+1)} & =\underset{\vartheta}{\arg \min }\left(\gamma_{1}\|\vartheta\|_{1}-\left\langle\xi_{1}, \vartheta-\nabla u\right\rangle+\frac{\mu_{1}}{2}\|\vartheta-\nabla u\|_{2}^{2}\right)= \\
& =\underset{d}{\arg \min }\left(\gamma_{1}\|\vartheta\|_{1}+\frac{\mu_{1}}{2}\left\|\vartheta-\nabla u^{(k+1)}-\frac{\xi_{1}^{(k)}}{\eta_{1}}\right\|_{2}^{2}\right) ; \\
\omega^{(k+1)} & =\underset{\omega}{\arg \min }\left(\gamma_{2}\|\omega\|_{1}-\left\langle\xi_{2}^{(k)}, \omega-\nabla^{\alpha} u^{(k+1)}\right\rangle+\frac{\mu_{2}}{2}\left\|\omega-\nabla^{\alpha} u^{(k+1)}\right\|_{2}^{2}\right)= \\
& =\underset{\omega}{\arg \min }\left(\gamma_{2}\|\omega\|_{1}+\frac{\mu_{2}}{2}\left\|\omega-\nabla^{\alpha} u^{(k+1)}-\frac{\xi_{2}^{(k)}}{\eta_{2}}\right\|_{2}^{2}\right) .
\end{aligned}
$$

The generalized shrinkage formula can be employed for solving the $\vartheta$ and $\omega$ subproblems as follows:

$$
\begin{aligned}
& \vartheta^{(k+1)}=\frac{\nabla u^{(k+1)}+\frac{\xi_{1}^{(k)}}{\mu_{1}}}{\left|\nabla u^{(k+1)}+\frac{\xi_{1}^{(k)}}{\mu_{1}}\right|} \cdot \max \left(\left|\nabla u^{(k+1)}+\frac{\xi_{1}^{(k)}}{\mu_{1}}\right|-\frac{\gamma_{1}}{\mu_{1}}, 0\right), \\
& \omega^{(k+1)}=\frac{\nabla^{\alpha} u^{(k+1)}+\frac{\xi_{2}^{(k)}}{\mu_{2}}}{\left|\nabla^{\alpha} u^{(k+1)}+\frac{\xi_{2}^{(k)}}{\mu_{2}}\right|} \cdot \max \left(\left|\nabla^{\alpha} u^{(k+1)}+\frac{\xi_{2}^{(k)}}{\mu_{2}}\right|-\frac{\gamma_{2}}{\mu_{2}}, 0\right) .
\end{aligned}
$$

The complete method is summarized in Algorithm 1. For the stopping condition, we end the loop if the maximum number of iterations $N$ has been carried out or the following condition is satisfied for prescribed tolerance $\varsigma$ :

$$
\frac{\left\|u^{(k)}-u^{(k-1)}\right\|_{2}}{\left\|u^{(k)}\right\|_{2}}<\varsigma,
$$

where $\varsigma$ is a small positive parameter. 


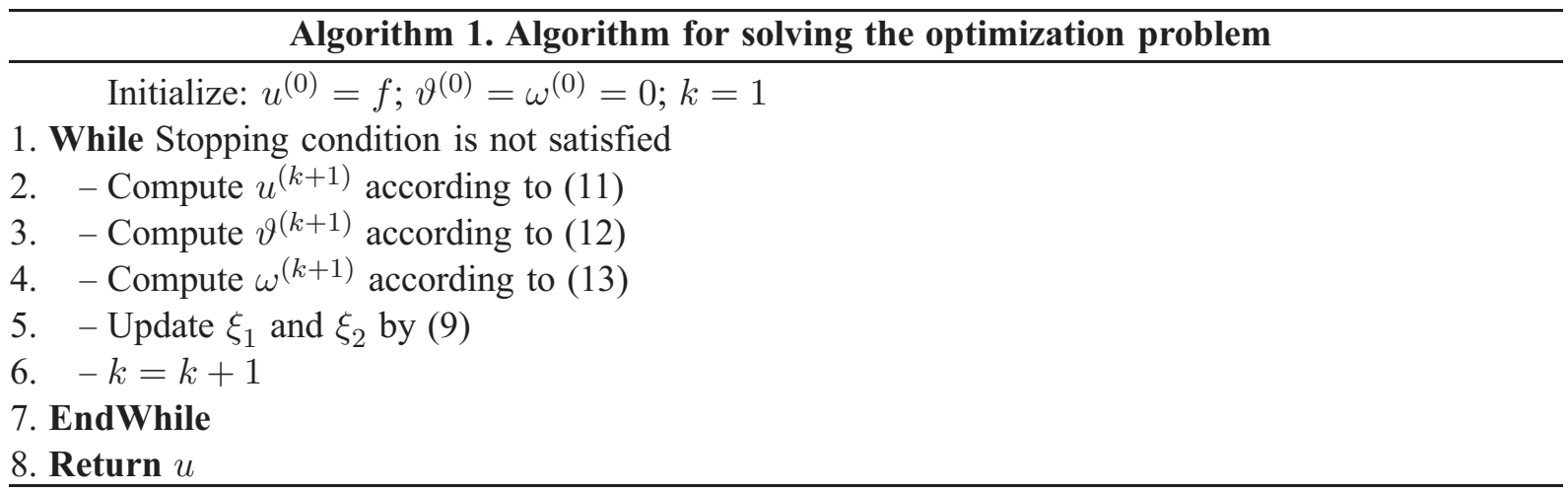

\section{Numerical experiments}

In this section, we present some numerical results for Poisson noise removal obtained by our proposed method. All experiments were carried out in Windows 10 and Matlab running on a desktop equipped with an Intel Core i3, $2.1 \mathrm{GHz}$ and $12 \mathrm{~GB}$ of RAM. In experiments, we compare our method with known methods for Poisson noise removal using total variation model (TVM) shown in (1), and fractional-order total variation model (FTVM) shown in (2). The compared models are implemented by the state-of-the-art alternating minimization algorithm. The original test images are shown in Figure 1.

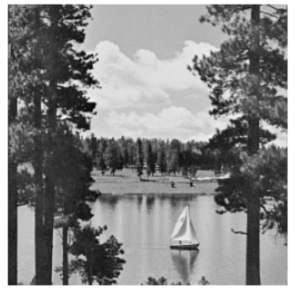

(a) Lake

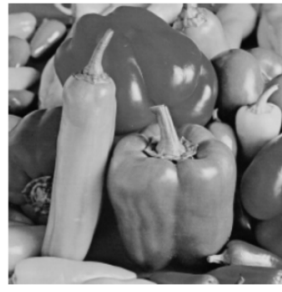

(b) Peppers

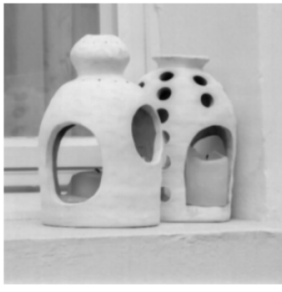

(c) Lamp

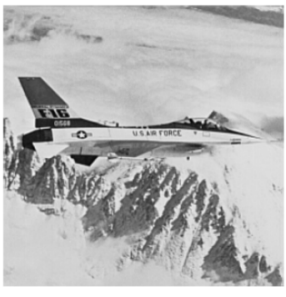

(d) Airplane

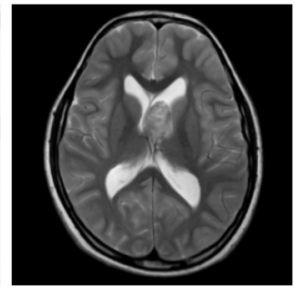

(e) Brain

Fig. 1. Test images

For assessing the quality of restoration results with compared methods, we use peak signal-tonoise ratio (PSNR) and Structural Similarity Index (SSIM) defined as follows [Bovik, 2006]:

$$
\begin{gathered}
P S N R=10 \log _{10}\left(\frac{255^{2} \cdot M N}{\left\|u^{*}-u\right\|_{2}^{2}}\right), \\
\operatorname{SSIM}\left(u, u^{*}\right)=\frac{\left(2 \mu_{u} \mu_{u^{*}}+c_{1}\right)\left(2 \sigma_{u, u^{*}}+c_{2}\right)}{\left(\mu_{u}^{2}+\mu_{u^{*}}^{2}+c_{1}\right)\left(\sigma_{u}^{2}+\sigma_{u^{*}}^{2}+c_{2}\right)},
\end{gathered}
$$

where $M$ and $N$ are the number of image pixels in rows and columns; $u, u^{*}$ are the original image, the reconstructed or noisy image accordingly $\mu_{u}, \mu_{u^{*}}$ are the means of $u, u^{*}$ respectively; $\sigma_{u}, \sigma_{u^{*}}$ their standard deviations; $\sigma_{u, u^{*}}$ the covariance of two images $u$ and $u^{*} ; c_{1}=\left(K_{1} L\right)^{2} ; c_{2}=\left(K_{2} L\right)^{2} ; L$ is the dynamic range of the pixel values (255 for 8-bit grayscale images); and finally $K_{1} \ll 1, K_{2} \ll 1$ are small constants.

For our experiments, we set tolerance in (14) $\varsigma=0,00004$ and $N=500$. The observed images in our experiments are simulated as follows. To test different noise levels, the noisy images are generated by Poisson noise with some fixed value Peak. Empirically, all of the compared methods perform image denoising with their optimal parameters. All images are processed with the equivalent parameters $\gamma_{1}=$ $=0,6, \gamma_{2}=0,4$, which gave the best restoration results. For our models, we set empirically $\mu_{1}=0,01$, $\mu_{2}=0,01$, and $\alpha=1,6$. 
In Figures 2 and 3 we show the results of compared methods for noise levels Peak $=120$. In Figures 2, $a$ and 3, a, we show the original images. In Figures 2, $b$ and 3, $b$ we show the noisy images. In the others Figures 2, $c-e$ and Figures 3, $c-e$ we show respectively the reconstructions given by TVM, FTVM, and proposed model.

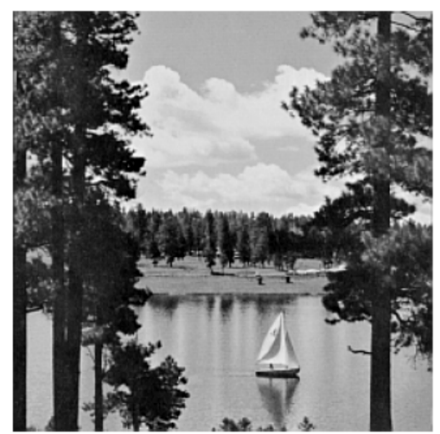

(a)

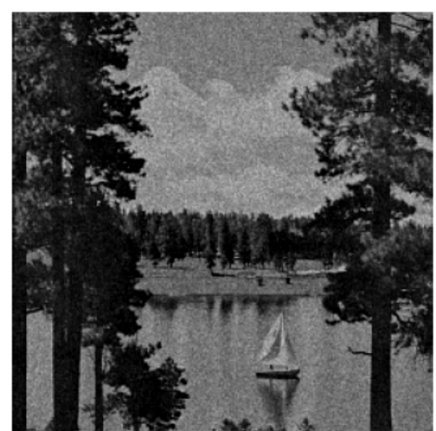

(b)

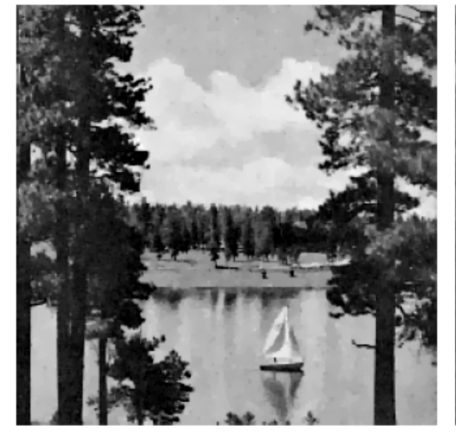

(c)

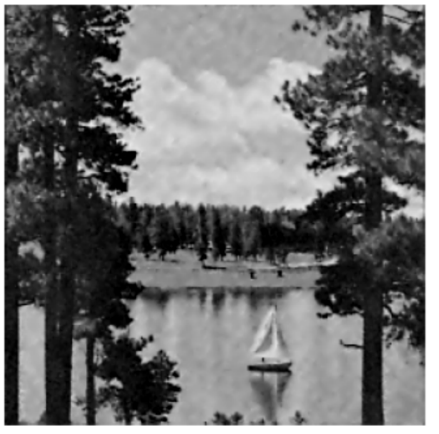

(d)

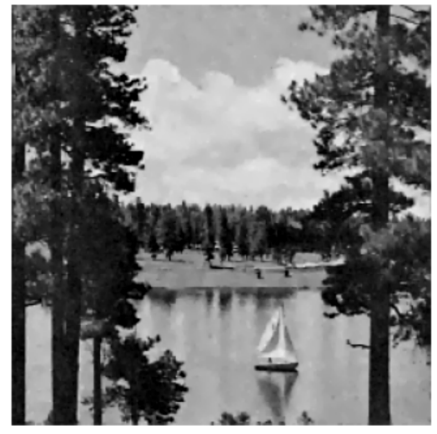

(e)

Fig. 2. Results for image «Lake»: recovered images of different approaches for image denoising: a) original image; b) noisy image $f$ with Peak $=120$; c) restored image by TVM; d) restored image by FTVM; e) restored image by our approach

In Figures 4 and 5 we show the results details of compared methods for noise levels Peak= $=120$. In Figures 4, $a$ and 5, $a$ we represent the details of original image. In Figures 4, $b$ and 5, $b$ we represent the details of noisy images, while in the others Figures 4, $c-e$ and Figures 5, $c-e$ we show respectively the reconstructions given by TVM, FTVM, and our proposed model. It can be seen that our method gives even better visual improvement than the other two methods. For the comparison of the performance quantitatively, we report the PSNR and SSIM values in Table 1.

In Figure 6 we exhibit the results of compared methods for noise levels Peak $=60$. In first column $6, a$ we also represent the original images, while in second column $6, b$ we show the noisy image. In columns $6, c-e$ we show reconstructions given by TVM, FTVM, and our proposed model.

In Figures 7, $a$ we represent the details of original image. In Figures 7, $b$ we represent the details of noisy images, while in the others, in Figures $7, c-e$ we show respectively the reconstructions given by TVM, FTVM, and our proposed model. The measures of PSNR and SSIM values are also reported in Table 2.

Finally in Figure 8 we show the results of compared methods for noise levels Peak $=20$. In first column Figure 8, $a$ we also represent the original images, while in second column Figure $8, b$ we show the noisy image. In columns Figure $8, c-e$ we show reconstructions given by TVM, FTVM, and our proposed model. Meanwhile in Figure 9 we show the zoomed-in part of the recovered images in Figure 8. The measures of PSNR and SSIM values are also reported in Table 3. 


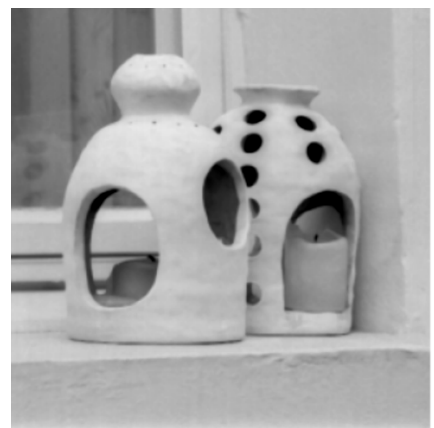

(a)

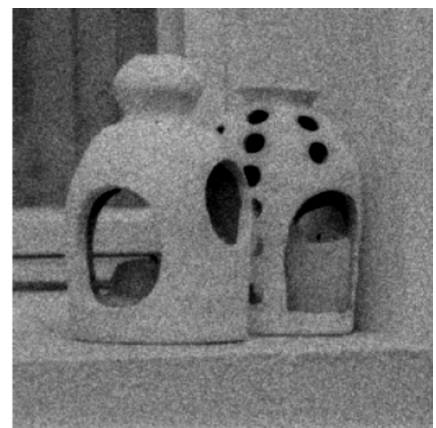

(b)

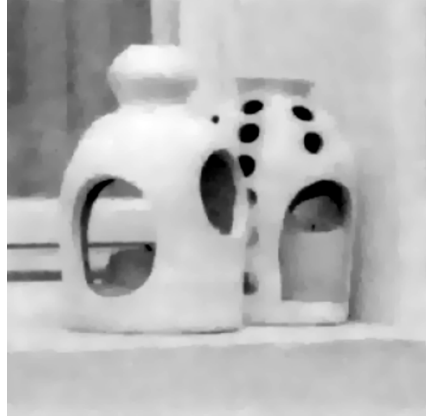

(c)

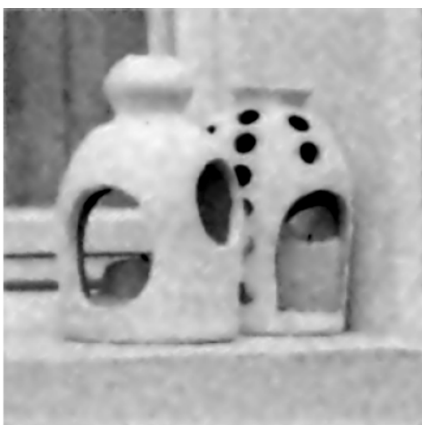

(d)

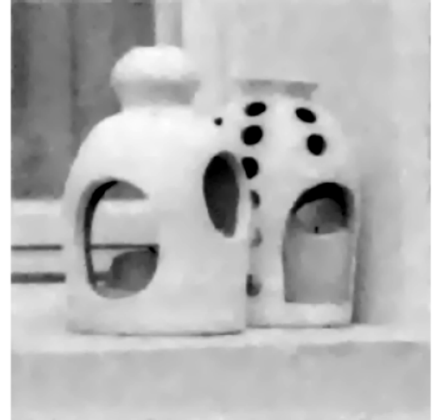

(e)

Fig. 3. Results for image «Lamp»: recovered images of different approaches for image denoising: a) original image; b) noisy image $f$ with Peak $=120$; c) restored image by TVM; d) restored image by FTVM; e) restored image by our approach

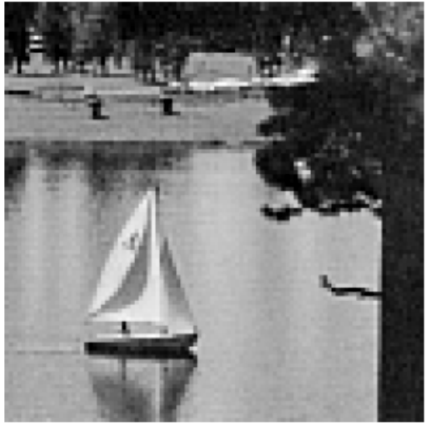

(a)

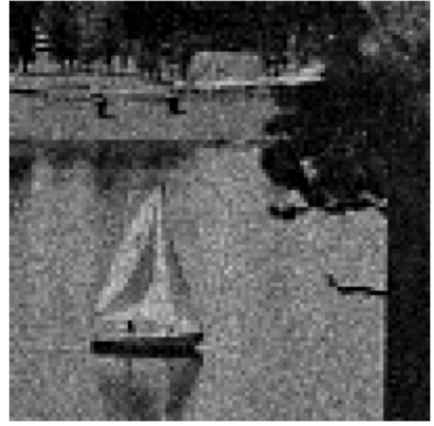

(b)

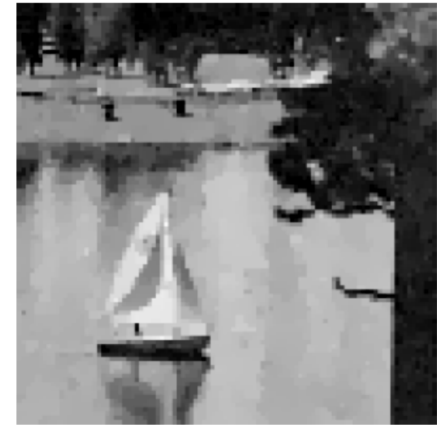

(c)

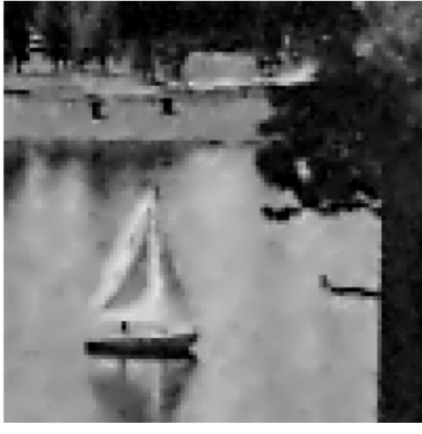

(d)

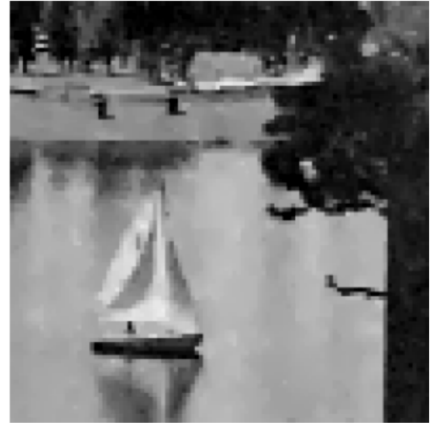

(e)

Fig. 4. The zoomed-in part of the recovered images in Figure 2: a) original image; b) noisy image $f$ with $P e a k=$ $=120$; c) restored image by TVM; d) restored image by FTVM; e) restore images by our approach 


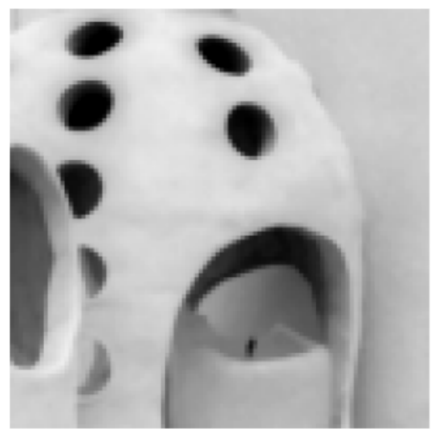

(a)

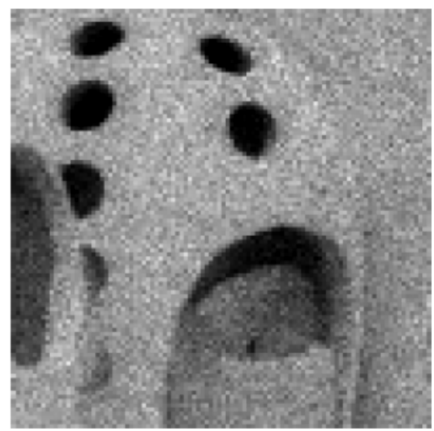

(b)

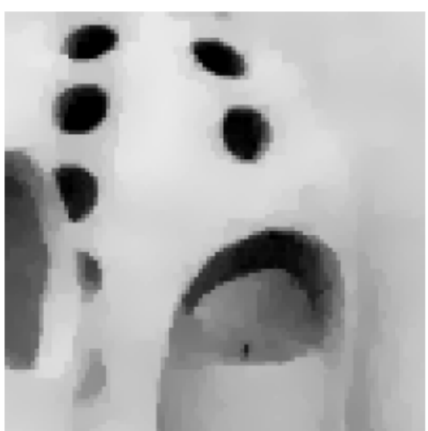

(c)

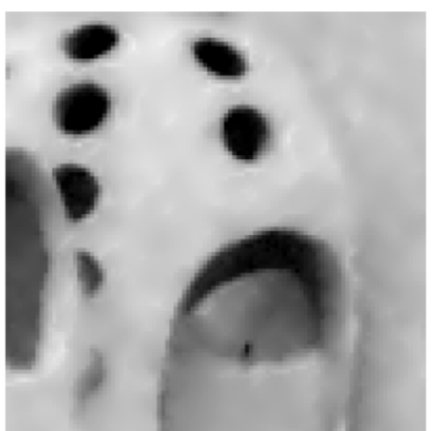

(d)

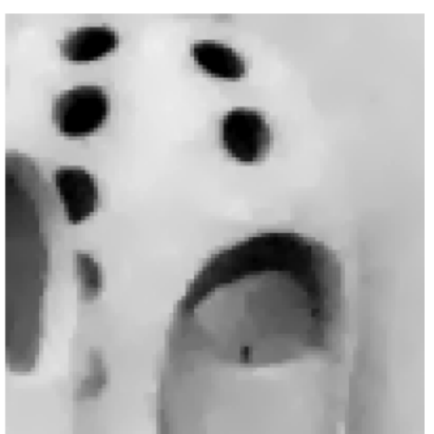

(e)

Fig. 5. The zoomed-in part of the recovered images in Figure 3: a) original image; b) noisy image $f$ with Peak= $=120$; c) restored image by TVM; d) restored image by FTVM; e) restored image by our approach
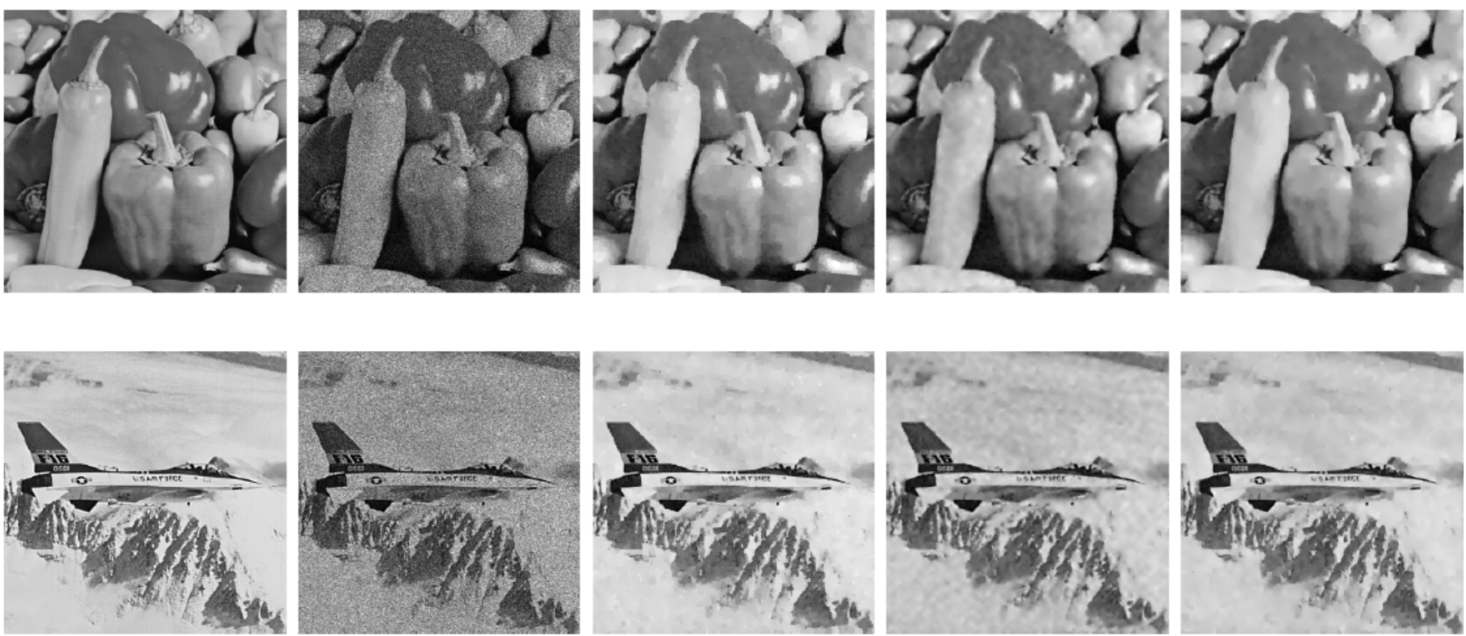

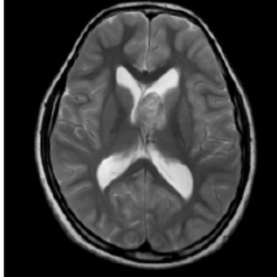

(a)

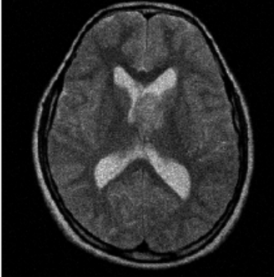

(b)

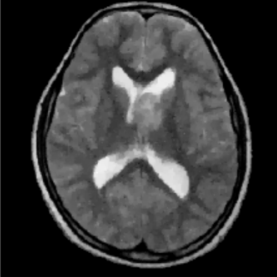

(c)

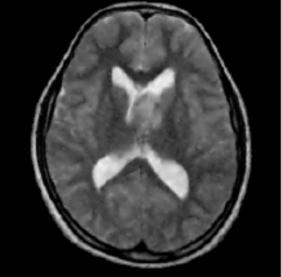

(d)

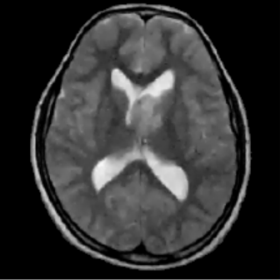

(e)

Fig. 6. Recovered results for the test images with noise level Peak=60: a) original images; b) noisy images $f$ with Peak $=60 ; \mathrm{c}$ ) restored images by TVM; d) restored images by FTVM; e) restored images by our approach 

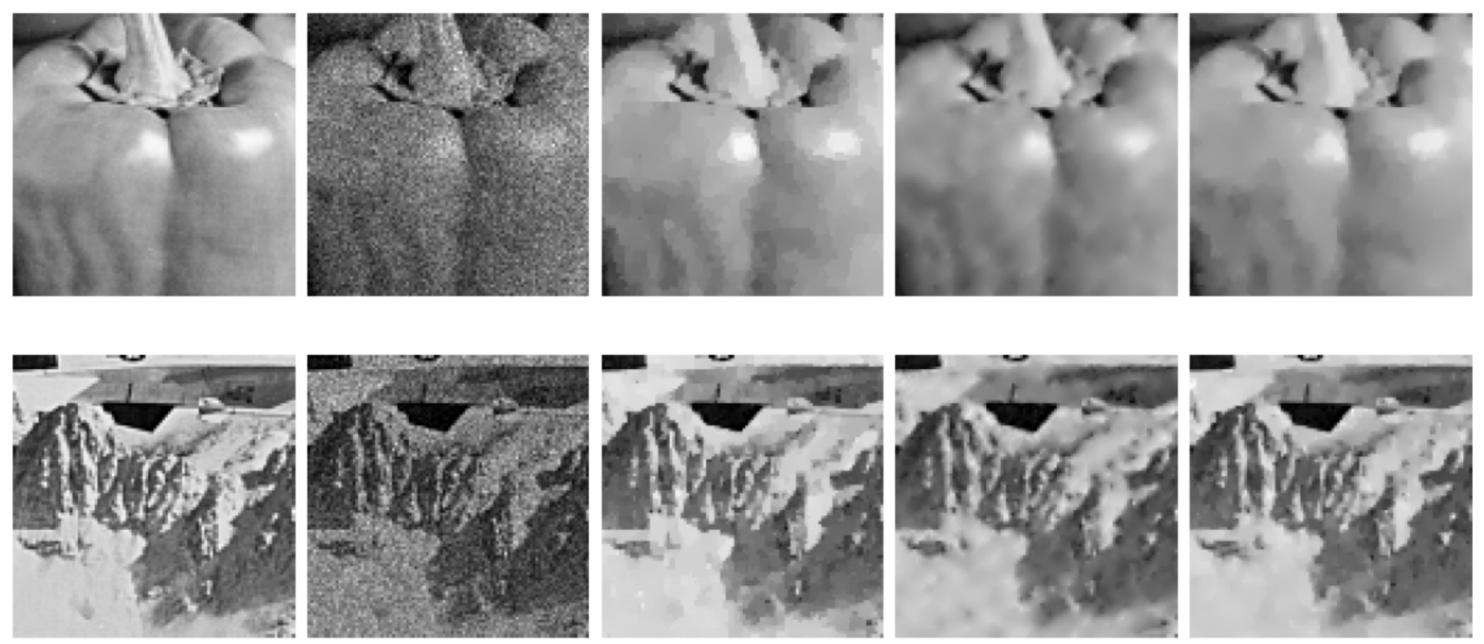

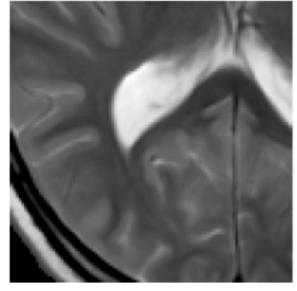

(a)

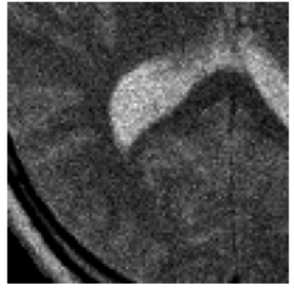

(b)

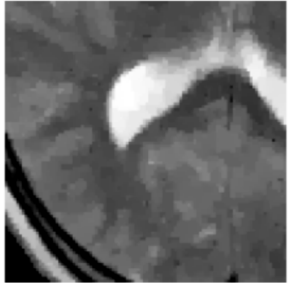

(c)

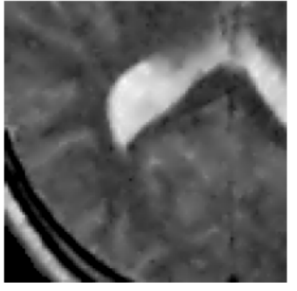

(d)

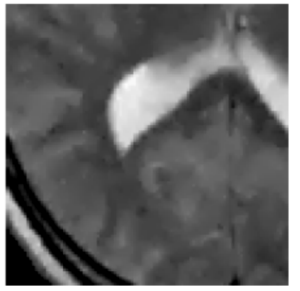

(e)

Fig. 7. The zoomed-in part of the recovered images in Figure 6: a) original images; b) noisy images $f$ with Peak $=60 ; \mathrm{c}$ ) restored images by TVM; d) restored images by FTVM; e) restored images by our approach
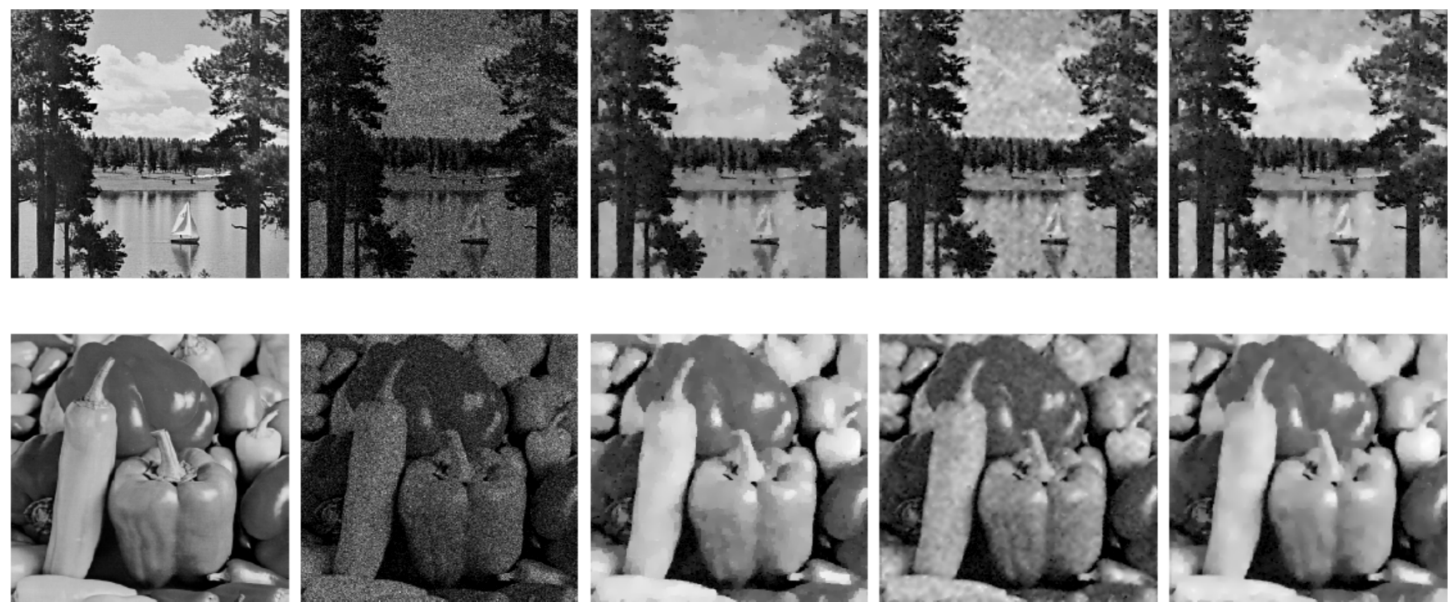

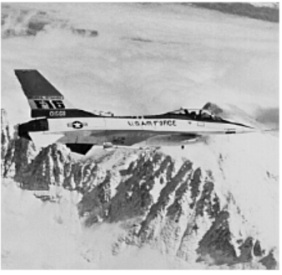

(a)

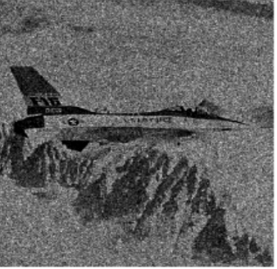

(b)

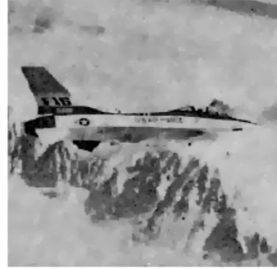

(c)

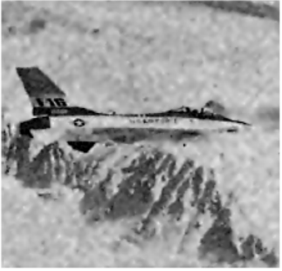

(d)

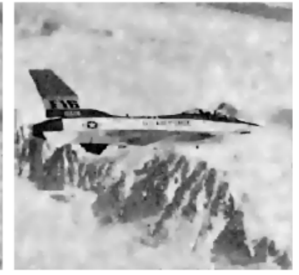

(e)

Fig. 8. Recovered results for the test images with noise level Peak = 20: a) original images; b) noisy images $f$ with $P e a k=20 ;$ c) restored images by TVM; d) restored images by FTVM; e) restored images by our approach 
Table 1. PSNR and SSIM values for noisy images and restored images with noise level Peak $=120$

\begin{tabular}{|c|c|c|c|c|c|c|c|c|}
\hline \multirow{2}{*}{ Image } & \multicolumn{5}{|c|}{ PSNR } & \multicolumn{4}{c|}{ SSIM } \\
\cline { 2 - 9 } & Noisy & TVM & FTVM & Ours & Noisy & TVM & FTVM & Ours \\
\hline Lake & 23,8684 & 27,4118 & 27,4606 & 28,1593 & 0,6794 & 0,8543 & 0,8495 & 0,8659 \\
\hline Peppers & 23,5030 & 30,1413 & 30,3140 & 30,9169 & 0,5707 & 0,8802 & 0,8952 & 0,9051 \\
\hline Lamp & 21,9740 & 32,9495 & 32,7573 & 33,5627 & 0,3505 & 0,9189 & 0,8916 & 0,9276 \\
\hline Airplane & 22,3381 & 26,9513 & 27,4429 & 27,8502 & 0,5129 & 0,8279 & 0,8231 & 0,8438 \\
\hline Brain & 26,9486 & 30,8859 & 31,3027 & 31,9143 & 0,7873 & 0,9174 & 0,9216 & 0,9332 \\
\hline \hline Average & 23,7264 & 29,6680 & 29,8555 & 30,4807 & 0,5802 & 0,8797 & 0,8762 & 0,8951 \\
\hline
\end{tabular}

Table 2. PSNR and SSIM values for noisy images and restored images with noise level Peak $=60$

\begin{tabular}{|c|c|c|c|c|c|c|c|c|}
\hline \multirow{2}{*}{ Image } & \multicolumn{4}{|c|}{ PSNR } & \multicolumn{4}{c|}{ SSIM } \\
\cline { 2 - 9 } & Noisy & TVM & FTVM & Ours & Noisy & TVM & FTVM & Ours \\
\hline Lake & 20,8402 & 25,3176 & 25,4088 & 25,5867 & 0,5733 & 0,8171 & 0,7956 & 0,8224 \\
\hline Peppers & 20,5111 & 28,1191 & 28,4811 & 29,0469 & 0,4619 & 0,8421 & 0,8655 & 0,8742 \\
\hline Lamp & 18,9986 & 31,3696 & 30,8046 & 31,9269 & 0,2634 & 0,8953 & 0,8704 & 0,9101 \\
\hline Airplane & 19,2591 & 25,9806 & 25,9168 & 26,5753 & 0,4136 & 0,8003 & 0,7680 & 0,8070 \\
\hline Brain & 24,0254 & 29,6099 & 29,98667 & 30,6999 & 0,7133 & 0,8900 & 0,9048 & 0,9123 \\
\hline \hline Average & 20,7269 & 28,0794 & 28,1196 & 28,7671 & 0,4851 & 0,8490 & 0,8409 & 0,8652 \\
\hline
\end{tabular}
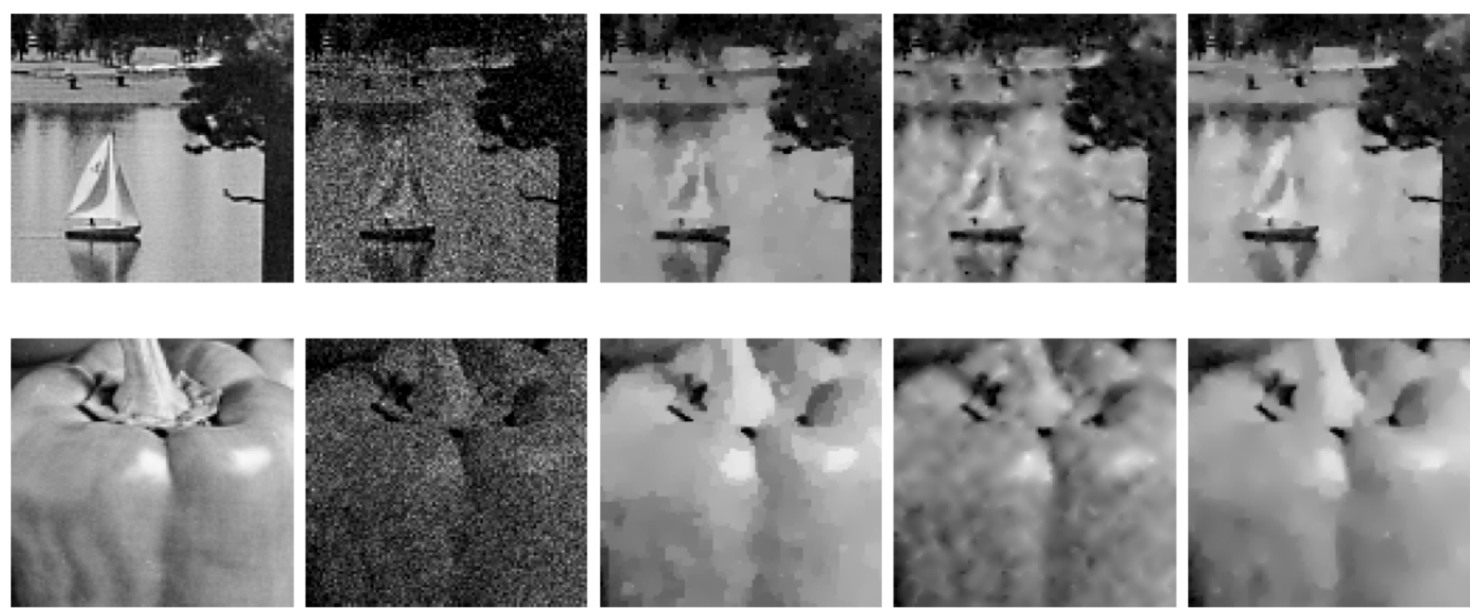

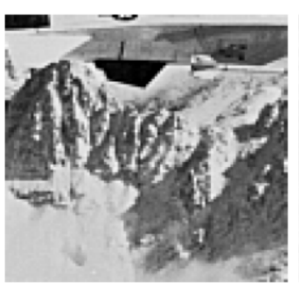

(a)

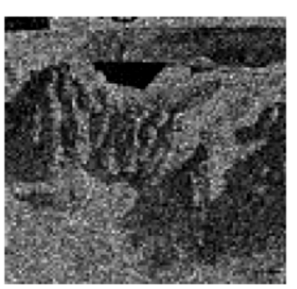

(b)

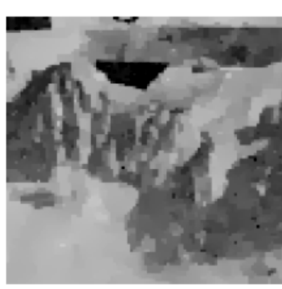

(c)

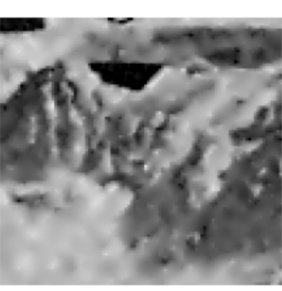

(d)

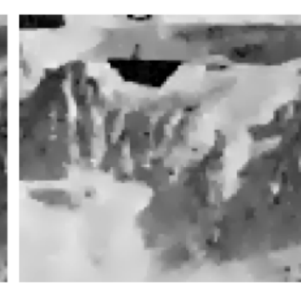

(e)

Fig. 9. The zoomed-in part of the recovered images in Figure (8): a) original images; b) noisy images $f$ with Peak $=20 ; \mathrm{c}$ ) restored images by TVM; d) restored images by FTVM; e) restored images by our approach

From Figures we can see that the images recovered by our proposed model are better quality than those of the compared approaches. Beside the measurable comparisons reported in Tables 1-3, the our 
Table 3. PSNR and SSIM values for noisy images and restored images with noise level Peak $=20$

\begin{tabular}{|c|c|c|c|c|c|c|c|c|}
\hline \multirow{2}{*}{ Image } & \multicolumn{4}{|c|}{ PSNR } & \multicolumn{4}{c|}{ SSIM } \\
\cline { 2 - 9 } & Noisy & TVM & FTVM & Ours & Noisy & TVM & FTVM & Ours \\
\hline Lake & 16,0928 & 22,4960 & 22,2765 & 22,8623 & 0,3975 & 0,6991 & 0,6641 & 0,7170 \\
\hline Peppers & 15,7160 & 25,7891 & 25,5059 & 26,2656 & 0,3007 & 0,7772 & 0,7498 & 0,8291 \\
\hline Lamp & 14,1413 & 28,0472 & 27,0481 & 28,8260 & 0,1538 & 0,8330 & 0,7367 & 0,8615 \\
\hline Airplane & 14,5166 & 23,5288 & 22,8528 & 23,9178 & 0,2727 & 0,6470 & 0,7149 & 0,7227 \\
\hline Brain & 19,6973 & 26,6947 & 26,9622 & 27,4305 & 0,6047 & 0,8114 & 0,8424 & 0,8663 \\
\hline \hline Average & 16,0328 & 25,3112 & 24,9291 & 25,8605 & 0,3459 & 0,7535 & 0,7416 & 0,7993 \\
\hline
\end{tabular}

proposed approach gets higher PSNR, SSIM values than those of the TVM and FTVM approaches on all images. It indicates the competitive superiority of the proposed method for Poisson noise removal.

\section{Conclusions}

In this paper, we have researched the hybrid regularizers model, combining the first order and fractional-order TV for denoising image corrupted by Poisson noise. Computationally, an improved highly efficient alternating minimization algorithm is employed for solving the proposed optimization problem. Finally, compared with several existing state-of-the-art approaches, the experiments demonstrate the superiority of the proposed method for suppressing Poisson noise.

\section{References}

Aubert G., Kornprobst P. Mathematical Problems in Image Processing: partial differential equations and the calculus of variations // Applied Mathematical Science. - Vol. 147, 2 ed. - New York: Springer-Verlag, 2006. $-379 \mathrm{p}$.

Bardsley J.M., Goldes J. Techniques for regularization parameter and hyper-parameter selection in PET and SPECT imaging // Inverse Probl. Sci. En. - 2011. - Vol. 19, no. 2. - P. 267-280.

Bovik A.C., Wang Z. Modern Image Quality Assessment, Synthesis Lectures on Image, Video, and Multimedia Processing. - Morgan and Claypool Publishers, 2006. - 156 p.

Bredies K., Kunisch K., Pock T. Total generalized variation // SIAM J. Imaging Sci. - 2010. - Vol. 3, no. 3. - P. 492-526.

Chambolle A. An algorithm for total variation minimization and applications // J. Math. Imaging Vis. 2004. - Vol. 20. - P. 89-97.

Chan R. H., Liang H. et al. High-order total variation regularization approach for axially symmetric object tomography from a single radiograph // Inverse Problems and Imaging. - 2015. - Vol. 9, no. $1 .-$ P. $55-77$.

Chen D., Chen Y.Q., Xue D. Fractional-order total variation image denoising based on proximity algorithm // Appl. Math. Comput. - 2015. - Vol. 257. - P. 537-545.

Chowdhury M. R., Zhang J., Qin J., Lou Y. Poisson image denoising based on fractional-order total variation // Inverse Problems and Imaging. - 2020. - Vol. 14, no. 1. - P. 77-96.

Dong $F$., Chen $Y$. A fractional-order derivative based variational framework for image denoising // Inverse Probl. Imaging. - 2016. - Vol. 10. - P. 27-50.

Frosioa I., Borghese N. A. Compression and smart coding of offset and gain maps for intraoral digital x-ray sensors // Med. Phys. - 2009. - Vol. 36, no. 2. - P. 464-479.

Goldstein T., Osher S. The split Bregman method for L1-regularized problems // SIAM J. Imaging Sci. - 2009. - Vol. 2, no. 2. - P. 89-97. 
He С., Ни С. et. al. An Adaptive Total Generalized Variation Model with Augmented Lagrangian Method for Image Denoising // Mathematical Problems in Engineering. - 2014. - Vol. 2014. Article ID 157893. - $11 \mathrm{p}$.

He C., Hu C., Zhang W., Shi B. A Fast Adaptive Parameter Estimation for Total Variation Image Restoration // IEEE Trans. Image Process. - 2014. - Vol. 23, no. 12. - P. 4954-4967.

Huang Y.M., Ng M. K., Wen Y.W. A fast total variation minimization method for image restoration // Multiscale Model. Sim. - 2008. - Vol. 7, no. 2. - P. 774-795.

Jiang L., Huang J., Lv X. G., Liu J. Restoring Poissonian Images by a Combined First-Order and Second-Order Variation Approach // J. Math. - 2013. - Vol. 274573. - 11 p.

Kayyar S. H., Jidesh P. Non-local total variation regularization approach for image restoration under a Poisson degradation // Journal of Modern Optics. - 2018. - Vol. 65. - P. 2231-2242.

Khan M.A., Chen W., Ullah A. et al. A mesh-free algorithm for ROF model // EURASIP Journal on Advances in Signal Processing. - 2017. - Vol. 2017, no. 1. - Article ID 53. - 16 p.

Lanza A., Morigi S., Sgallari F., Wen Y.W. Image restoration with Poisson-Gaussian mixed noise // Computer Methods in Biomechanics and Biomedical Engineering: Imaging and Visualization. 2014. - Vol. 2, no. 1. - P. 12-24.

Le T., Chartrand R., Asaki T.J. A variational approach to reconstructing images corrupted by Poisson noise // Journal of Mathematical Imaging and Vision. - 2007. - Vol. 27. - P. 257-263.

Li J., Shen Z., Yin R., Zhang X. A reweighted method for image restoration with Poisson and mixed Poisson - Gaussian noise // Inverse Problems \& Imaging. - 2015. - Vol. 9, no. 3. - P. 875-894.

Liu $X$. Augmented Lagrangian method for total generalized variation based Poissonian image restoration // Computers \& Mathematics with Applications. - 2016. - Vol. 71, no. 8. - P. 1694 1705.

Lysaker M., Lundervold A., Tai X.C. Noise removal using fourth order partial differential equation with applications to medical magnetic resonance images in space and time // IEEE Trans. Image Process. - 2003. - Vol. 12. - P. 1579-1590.

Myllykoski M., Glowinski R., Karkkainen T., Rossi T. A New Augmented Lagrangian Approach for $L^{1}$-mean Curvature Image Denoising // SIAM J. Imaging Sci. - 2015. - Vol. 8, no. 1. - P. 95125.

Papafitsoros K., Schönlieb C. B. A Combined First and Second Order Variational Approach for Image Reconstruction // J. Math. Imaging Vis. - 2014. - Vol. 48. - P. 308-338.

Pham C. T., Tran T. T. T., Nguyen T. C., Vo D. H. Second-order total generalized variation based model for restoring images with mixed Poisson-Gaussian noise // Inf. and Control Sys. - 2021. Vol. 2. - P. 20-32.

Pham C.T., Tran T.T.T., Gamard G. An efficient total variation minimization method for image restoration // Informatica. - 2020. - Vol. 31, no. 3. - P. 539-560.

Pham C.T. et al. An algorithm for image restoration with mixed noise using total variation regularization // Cybern. Phys. - 2019. - Vol. 8, no. 2. - P. 73-82.

Pham C. T., Kopylov A. V. Tree-serial parametric dynamic programming with flexible prior model for image denoising // Comput. Opt. - 2018. - Vol. 42, no. 5. - P. 838-845.

Pham C. T., Gamard G., Kopylov A., Tran T. T. T. An algorithm for image restoration with mixed noise using total variation regularization // Turk. J. Elec. Eng. \& Comp. Sci. - 2018. - Vol. 26, no. 6. P. 2831-2845.

Pham C. T., Kopylov A. Multi-quadratic dynamic programming procedure of edge-preserving denoising for medical images // Int. Arch. Photogramm. - 2015. - Vol. XL-5-W6. - P. 101-106.

Sarder P., Nehorai A. Deconvolution method for 3D fluorescence microscopy images // IEEE Signal Process. Magazine. - 2006. - Vol. 23, no. 3. - P. 32-45. 
Setzer S., Steidl G., Teuber T. Deblurring Poissonian images by split Bregman techniques // Journal of Visual Communication and Image Representation. - 2010. - Vol. 21, no. 3. - P. 193-199.

Yang Y., Zhao D. An Adaptive Model Combining a Total Variation Filter and a Fractional-Order Filter for Image Restoration // Journal of Algorithms \& Computational Technology. - 2019. - P. 1-11.

Zhang J., Wei Z., Xiao L. Adaptive fractional-order multi-scale method for image denoising // J. Math. Imaging Vision. - 2012. - Vol. 43. - P. 39-49.

Zhang J., Chen K. A total fractional-order variation model for image restoration with nonhomogeneous boundary conditions and its numerical solution // SIAM J. Imaging Sci. - 2015. - Vol. 8. P. 2487-2518.

Zhang J., Ma M., Wu Z., and Deng C. High-Order Total Bounded Variation Model and Its Fast Algorithm for Poissonian Image Restoration // Math. Probl. Eng. - 2019. - Vol. 2019. - Article ID 2502731. - $11 \mathrm{p}$.

Zhang J., Chen R., Deng C., Wang S. Fast linearized augmented Lagrangian method for Euler's elastica model // Numer. Math. Theory Methods Appl. - 2017. - Vol. 10. - P. 98-115.

Zhu W., Tai X.C., Chan T. A Fast Algorithm for a Mean Curvature Based Image Denoising Model Using Augmented Lagrangian Method // Efficient Algorithms for Global Optimization Methods in Computer Vision. Lecture Notes in Computer Science. - Berlin, Heidelberg: Springer, 2014. Vol. 8293. - P. 104-118.

Wang Y., Yang J., Yin W., Zhang Y. A New Alternating Minimization Algorithm for Total Variation Image Reconstruction // SIAM Journal on Imaging Sciences. - 2008. - Vol. 1, no. 3. - P. 248 272.

Wang X.D., Feng X.C., Wang W.W., Zhang W.J. Iterative reweighted total generalized variation based poisson noise removal model // Appl. Math. Comput. - 2013. - Vol. 223. - P. 264-277. 